\title{
Présences sonores. Musique, images et langue chez les Kuikuro du Haut Xingu
}

\section{Tommaso Montagnani}

\section{(2) OpenEdition}

1 Journals

Édition électronique

URL : https://journals.openedition.org/imagesrevues/493

DOI : 10.4000/imagesrevues.493

ISSN : 1778-3801

Éditeur :

Centre d'Histoire et Théorie des Arts, Groupe d'Anthropologie Historique de l'Occident Médiéval, Laboratoire d'Anthropologie Sociale, UMR 8210 Anthropologie et Histoire des Mondes Antiques

\section{(c) (7) \&}

Images Re-vues est mise à disposition selon les termes de la Licence Creative Commons Attribution Pas d'Utilisation Commerciale 4.0 International. 


\title{
PresenCES SONORES. \\ MusiQue, images et langue Chez les Kuikuro du HaUt Xingu
}

\author{
Tommaso Montagnani
}

L'objectif de cet article est de s'interroger sur le rôle des images mentales dans l'exécution et dans l'audition de la musique rituelle chez les Kuikuro du Haut Xingu. La musique instrumentale des flûtes sacrées Kagutu est considérée comme la voix des Itseke. Ces entités non-humaines font entendre leur nom à travers le son de la flûte et ils se manifestent ainsi auprès de l'auditoire sous forme de fragment mélodique. Afin de mémoriser les structures musicales complexes des suites jouées par ces flûtes sacrées, les musiciens ont recours à des moyens mnémotechniques impliquant des processus de visualisation qui guident aussi l'exécution rituelle. Les images ainsi visualisées se réfèrent à la fois à la structure interne de la pièce musicale et à la succession des suites pendant l'exécution du rituel Kagutu.

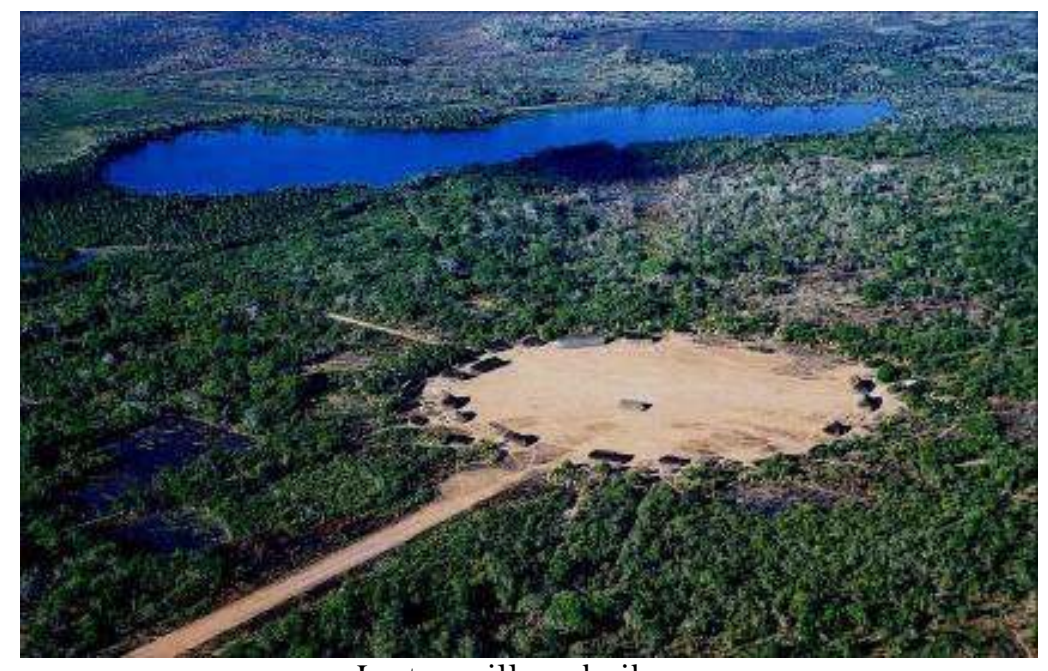

Ipatse, village kuikuro

Cet article ${ }^{1}$ a pour but de montrer le rôle des images, des gestes et du langage dans l'apprentissage et l'exécution de la musique kuikuro ${ }^{2}$.

La relation entre musique et langage dans le Haut Xingu a été traitée de façon remarquable par Rafael Menezes Bastos dans ses travaux sur la musique kamayura3. Cet

\footnotetext{
1 Les données présentées ici proviennent de deux voyages de terrain que j’ai effectués à Ipatse, le principal village kuikuro, entre 2008 et 2009, dans le cadre de la préparation d'une thèse sur la musique rituelle de ce groupe xinguanien.

${ }^{2}$ Les Kuikuro sont une population indigène d'Amazonie méridionale, de langue carib. Leurs villages se situent dans la partie sud-est du parc «Terra Indígena do Xingu », dans l'état du Mato Grosso, au Brésil. Les groupes qui habitent cette région, de langue arawak, carib, tupi et trumai, forment une constellation multi-ethnique et plurilingue d'une remarquable uniformité culturelle. Une partie importante des rituels xinguaniens possède donc un caractère inter-tribal. Le principal village kuikuro, Ipatse, se trouve entre le fleuve Kuluene et le fleuve Buriti, deux affluents du Xingu. Les villages xinguaniens, de forme circulaire, sont bâtis dans des espaces dégagés dans ce but dans la forêt basse qui caractérise cette région. Cette disposition circulaire des habitations règle le déroulement de la plupart des rituels, dont celui des flûtes sacrées Kagutu.
} 
auteur analyse la terminologie et le langage musicaux en se concentrant sur la façon dont les Kamayura parlent du son, des structures musicales et des différents actes de musiques. Par cette approche, il parvient à définir la pensée musicale des Kamayura à partir de leur propre discours et, en outre, à dépasser les limites de la discipline ethnomusicologique : son étude du langage musical s'insère ainsi dans le cadre plus large de l'anthropologie de la communication.

Le passage de la parole à la musique est aussi au cour du travail d'Anthony Seeger sur la musique des Suyá4, groupe n'appartenant pas à l'ensemble du Haut Xingu mais entretenant d'étroits rapports avec lui, et résidant dans le même parc. Le chercheur américain montre que dans la musique xinguanienne il n'existe souvent pas une limite nette entre le discours parlé, le chant et la musique instrumentale 5, et que c'est justement dans les formes intermédiaires que l'on peut observer la transformation du son en langage et du langage en musique. Ceci ne signifie pas que les Suyá n’opèrent pas de distinctions entre les genres : bien au contraire, ces distinctions existent pour eux comme pour les Kuikuro, et elles sont toujours rappelées par les musiciens. Mais ceux-ci développent néanmoins la capacité à reconnaître à tout moment la présence de la parole des esprits dans la musique instrumentale et l'écho des mélodies sacrées dans les chants profanes.

Dans cet article, je tenterai d'introduire un troisième agent dans la relation typiquement xinguanienne entre musique et langage: l'image. L'image de la musique kuikuro est à la fois la visualisation d'une forme qui correspond à la structure de la pièce à exécuter et l'image sonore d'une présence qui est évoquée dans la performance rituelle au moyen d'une musique instrumentale qui se fait langage.

\section{Pour une séquence de la figuration : image, musique et langage}

Chez les Kuikuro la musique est conçue comme une figuration sonore des esprits. Cette idée s'exprime particulièrement dans le rituel des flûtes sacrées appelées Kagutu, qui figurent parmi les instruments les plus prestigieux. Leur répertoire est extrêmement long et complexe, et très rares sont les musiciens qui parviennent à le maîtriser dans sa totalité. Cette élite peut ainsi assurer le déroulement du rituel à l'intérieur de l'espace qui lui est consacré, le Kuakutu, la maison des flûtes située sur la place du village, au cœur du cercle formé par les maisons. Lors de mon premier séjour, trois musiciens seulement connaissaient toutes les suites du répertoire kagutu : Jakalu, Kamangagü et Tupà. Ce dernier était très âgé et n'était plus en mesure de jouer sur ces flûtes en bois, grandes et lourdes. Il se souvenait néanmoins de la musique et pouvait chanter les suites sacrées. L'un de ses élèves, Tütekuegü, était alors à un stade très avancé de son apprentissage et connaissait la majorité des suites. Parmi les plus jeunes Kuikuro, seuls quelques-uns exprimaient le souhait de devenir un jour un musicien de Kagutu. Cela ne présage cependant pas d'une disparition définitive du savoir lié au rituel et aux musiques des flûtes sacrées (ce qui s'est produit chez d'autres groupes xinguaniens) car ce savoir a toujours été très confidentiel. Les récits des

\footnotetext{
3 Rafael J. de M. Bastos, A Musicológica Kamayurá : para uma antropologia da comunicação no Alto Xingu, Brasilia, FUNAI, 1978.

4. A. Seeger, Why Suya Sing : a musical anthropology of an Amazonian people, Cambridge, Cambridge University Press, 1987. Voir notamment le chapitre intitulé «From Speech to Song ».

5 Chez les Wauja, autre groupe xinguanien, les relations entre chant et musique instrumentale ont été observées par A. Camargo Piedade, O Canto do Kawoká. Thèse de Doctorat, Universidade Federal de Santa Catarina, 2004, et par M. I. C. Mello, «Relações de gênero e musicologia : reflexões para uma análise do contexto brasileiro ", Revista Eletrônica de Musicologia XI, 2007, p. 1; "The ritual of Iamurikuma and the Kawoká flutes ", dans Jonathan D. Hill et Jean-Pierre Chaumeil. (org.), Burst of Breath: New Research on Indigenous Ritual Wind Instruments in Lowland South America, University of Nebraska Press, 2008.
} 
plus anciens confirment que les musiciens de Kagutu n'étaient pas plus nombreux qu'aujourd'hui au moment de la création du Parc du Xingu6. Et la mythologie entérine ce statut d'un savoir réservé à une élite, puisque dans le mythe d'origine du rituel, Tãugi (le Soleil) explique aux hommes que la flûte Kagutu « ne sera pas jouée par tout le monde ${ }^{7}$.

Le son des flûtes sacrées reproduit dans le même temps les mélodies des esprits et leur voix. Musique et langage sont donc indissociables ici, comme dans une bonne partie de la musique kuikuro. Plus précisément, c'est un fragment mélodique précis qui assume, pour chaque pièce, la prononciation du nom de l'esprit qui en est l'énonciateur originel. Ce moment où la flûte parle littéralement rend perceptible la présence de l'esprit dans l'espace du village. Même si c'est ainsi essentiellement sur le terrain de l'audition que cette présence s'établit, les images occupent un grand rôle dans la mémorisation, l'apprentissage et l'exécution des suites sacrées. Les musiciens kuikuro visualisent mentalement la structure de la pièce à exécuter, conçue comme un parcours formé par un chemin principal et par des déviations latérales ou verticales. Ce parcours est utilisé comme un plan, une carte mentale qui guide l'exécution de l'interprète et qui est parfois dessinée sur le sol avec un bâton ou sur un papier, pendant la phase d'apprentissage ou au cours des entretiens avec les ethnographes, mais jamais pendant l'exécution rituelle. L'image est un guide, une partition de la pièce qui permet d'en reconstruire la structure grâce à un processus de représentation mentale des formes.

Le système musical kuikuro est donc basé sur la relation entre langue, son et image et sur l'interaction des trois. La dynamique de cette interaction peut être décrite par un schéma dans lequel l'image engendre la musique et la musique engendre le langage ; les trois éléments participent de façon complémentaire à la représentation de la présence (fig.1).

Fig.1.

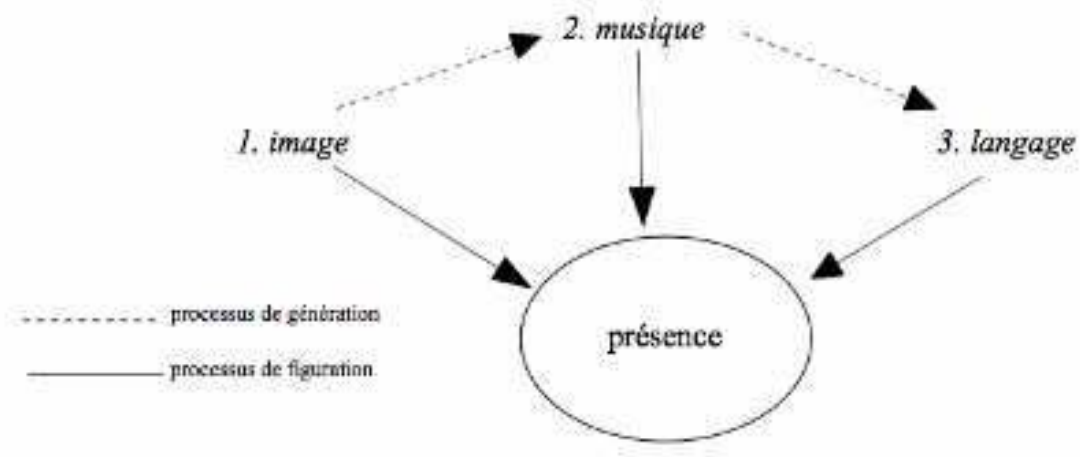

Dynamique d'interaction entre langue, son et image

Nous allons voir maintenant de quelle façon, dans le rituel Kagutu, l'image mentale d'un parcours est utilisée pour guider le choix des suites à exécuter pendant le rituel. Cette image est non seulement un parcours de la mémoire, mais aussi un support de la danse, puisque la chorégraphie des mouvements des musiciens est en partie basée sur le parcours mental des suites. Les termes kuikuro employés pour désigner l'exécution des musiques Kagutu semblent d'ailleurs indiquer non seulement l'importance attachée à l'émission sonore, mais aussi à la chorégraphie. Les Kuikuro utilisent non seulement le terme iki-jü (souffler), mais aussi celui de angune-nügü, forme transitive de angu (danser). L'expression angune-nügü Kagutu peut donc être traduite par «faire danser la flûte Kagutu » ${ }^{8}$. Il existe donc chez les Kuikuro non seulement une figuration des esprits au moyen d'une image

6 Sur le Parc du Xingu, voir B. Franchetto, M. Heckenbreger, Os Povos do Alto Xingu: História e cultura, Rio de Janeiro, UFRJ, 2001.

7 Cette information est extraite d'un récit mythique recueilli auprès du flûtiste Tütekuegü Kuikuro.

8 Je remercie Bruna Franchetto pour les informations linguistiques concernant les termes kuikuro ici employés. 
sonore, mais aussi une figuration de la musique elle-même: des formes visualisées mentalement correspondent à des structures musicales et à des séquences possibles de suites à exécuter pendant le rituel des flûtes sacrées.

\section{La voix des itseke dans le son de la flûte : figuration musicale des esprits}

L'impossibilité de voir est un trait essentiel du déroulement du rituel Kagutu. Cette absence de vision se manifeste à deux niveaux différents : les esprits (itseke) sont invisibles, et la flûte Kagutu ne peut pas être vue par les femmes. L'absence d'image à laquelle associer la musique semble donc être une caractéristique importante de ce rituel. Nous verrons qu'en réalité il existe bien des images dans ce rituel, mais qui ne sont pas reproduites : elles sont visualisées mentalement par les interprètes.

Les itseke sont les esprits de la cosmologie kuikuro. Ils sont très présents à la fois dans les mythes d'origines et dans la vie quotidienne de la communauté. Ils peuvent causer la maladie et la mort, mais ils peuvent également être invoqués par le chamane pendant certains rituels de guérison : ils l'aident à retrouver les fétiches cachés par les sorciers et qui provoquent toute sorte d'infortune. Le chamane peut aussi s'adresser directement aux esprits afin qu'ils cessent de rendre le patient malade. Les itseke sont des animaux/esprits ; ils ont donc les noms des animaux de la forêt et des poissons de la rivière.

Les itseke sont les 'propriétaires' et les auteurs des musiques sacrées Kagutu, raison pour laquelle ce répertoire est considéré comme le plus dangereux de toutes les formes de musique kuikuro. Une mauvaise exécution, des fautes ou des changements dans la mélodie peuvent provoquer la colère des esprits, qui se vengent en provoquant la maladie ou la mort du flûtiste. La menace qui pèse sur les musiciens, la pression qu'ils ressentent lors de la performance est une première forme de manifestation, sur le plan émotionnel, de la présence des esprits. Les émotions sont un vecteur de la perception de l'invisible; dans ce cas spécifique, la peur est l'émotion qui rend perceptible la présence de l'itseke.

Selon le mythe concernant l'origine de Kagutu, cette musique était jouée par les esprits dans leur village. Tãugi, le Soleil, était le maître de la cérémonie et les autres esprits participaient en chantant chacun leur propre pièce. Dans le mythe, les esprits chantent les pièces au lieu de jouer de la flûte. Au début de sa pièce, chaque esprit prononce son nom, comme s'il s'agissait d'une sorte de présentation. Cette situation se retrouve dans la performance rituelle: parmi les nombreuses suites Kagutu, celle intitulée Tolo Imitoho est composée de pièces nommées d'après les noms des esprits. Chaque pièce dans cette séquence a son maître, son propriétaire, qui est en réalité l'esprit qui la chantait dans le mythe d'origine, pendant l'exécution dirigée par Tãugi dans le village des itseke. Ce qui est raconté dans le mythe, c'est-à-dire la «présentation » des esprits au début de la pièce, se produit aussi lors du rituel : le début de la mélodie de chaque pièce est en fait le nom de l'esprit qui en est le propriétaire-auteur. La flûte est ainsi capable de reproduire la voix de l'esprit en train de prononcer son propre nom. L'esprit en ce sens reste invisible dans le rituel, sa présence est sonore.

Nous allons montrer ce phénomène à partir d'un extrait de la pièce Utigi (un poisson/esprit) de la suite Imitoho (fig.2). 
Fig.2.

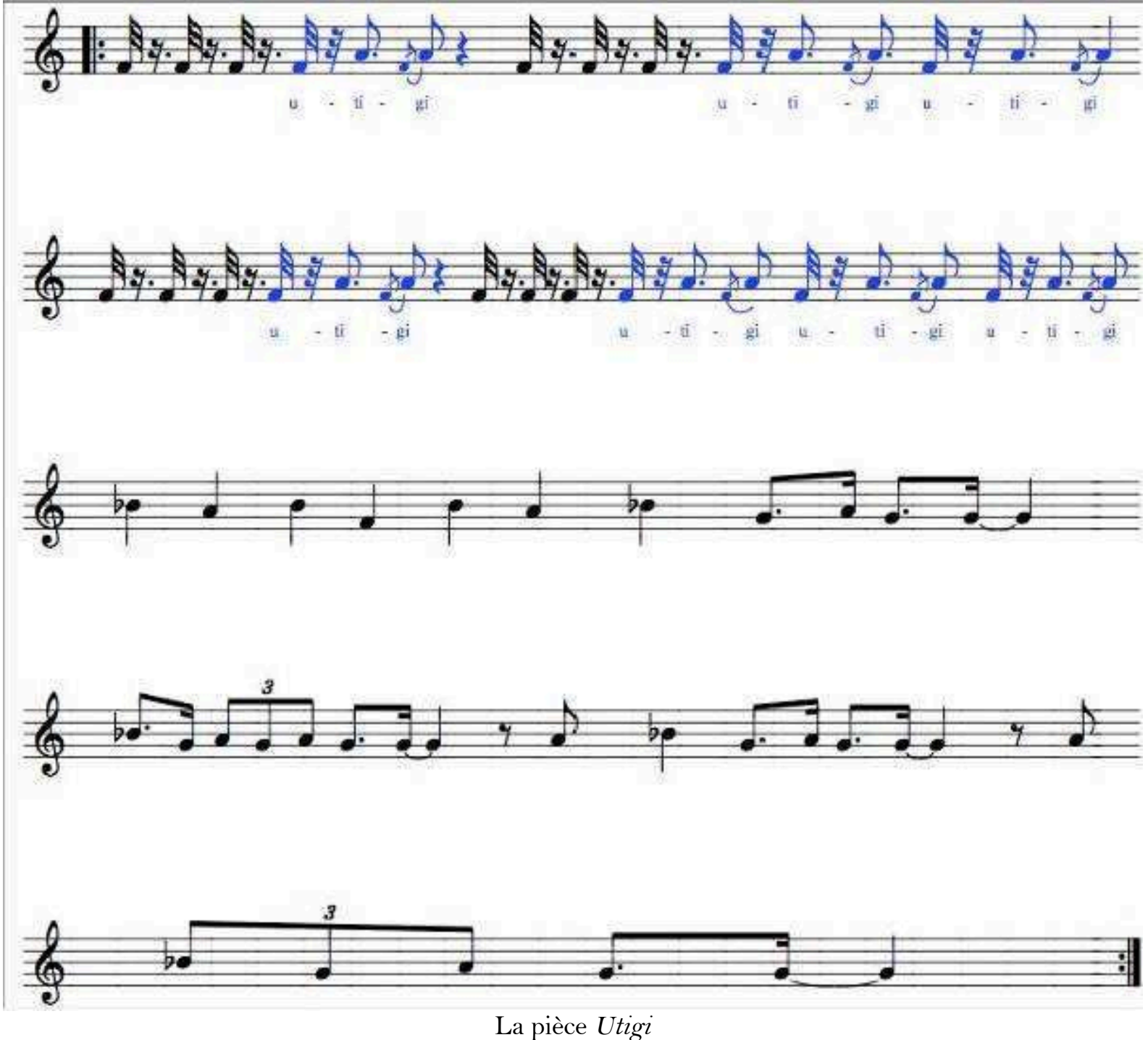

Les notes en bleu représentent les fragments de la mélodie où « la flûte dit le nom de l'esprit » selon la formule employée communément par les Kuikuro. (Audio.1 en Annexe) En l'occurrence cet esprit auteur de la pièce est Utigi. Par quels procédés l'instrument peut-il « parler », au point que les Kuikuro entendent dans le son de la flûte un nom propre?

Pour répondre à cette question il nous faut analyser le profil mélodique de la mélodie/nom et la durée des trois notes qui la composent. Dans le schéma qui suit, les numéros entre parenthèses représentent des unités minimales de durée: il s'agit là du concept de «mora $»^{10}$ emprunté à la linguistique et adapté à l'analyse musicale. Cette opération est justifiée par le fait que la mélodie en question est aussi un nom, donc un mot qui est entendu par les Kuikuro lorsqu'ils jouent ou écoutent ce fragment mélodique. Le but de cet emprunt est d'introduire l'analyse linguistique du mot «Utigi», tout en gardant la connexion fondamentale entre musique et langage qui caractérise le phénomène en question. Le profil mélodique du fragment que nous allons ainsi pouvoir observer sera ensuite comparé à l'analyse rythmique du nom de l'itseke qui lui est associé (fig.3).

9 Il s'agit de l'expression communément utilisée par les Kuikuro.

10 B. Hayes, Metrical Stress Theory. Principles and case studies, Chicago/Londres, University of Chicago Press, 1995. Une mora est une unité de poids de la syllabe. 
Fig.3.

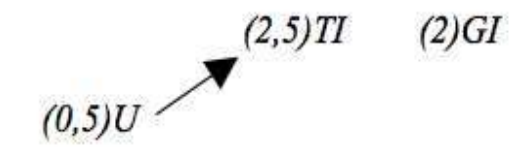

Profil mélodique du fragment Utigi

Les notes qui correspondent aux syllabes $\mathrm{Ti}$ et $\mathrm{Gi}$ sont plus aiguës que la première (syllabe U). La note de la syllabe Ti est la plus longue avec deux unités et demie (une croche pointée sur la partition), alors que celle qui correspond à la voyelle $U$ est la plus courte (une double croche). Le fait que le premier La (syllabe Ti) soit plus long est dû à l'accent tonique du nom Utigi, accent qui tombe justement sur cette syllabe. Les intervalles et les durées rythmiques de la mélodie de la flûte sont utilisés pour reproduire en musique la prosodie de la langue kuikuro.

En analysant le mot Utigi $i^{11}$ du point de vue de sa structure rythmique, on pourra observer un profil qui ressemble à celui du fragment mélodique qui lui est associé. Le $\mathrm{X}$ audessus de la syllabe $\boldsymbol{T} \boldsymbol{i}$ indique un pic d'intensité (fig.4).

Fig.4.

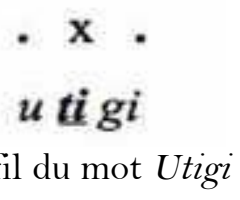

Cette comparaison permet de mettre en évidence certains principes sur lesquels l'imitation musicale de la langue kuikuro est basée: on remarquera que la différence d'intensité qui existe entre $\mathrm{U}$ et $\mathrm{Ti}$ est musicalement représentée à la fois par un intervalle ascendant d'une tierce majeure $(\mathrm{Fa}-\mathrm{La})$ et par une plus longue durée rythmique (une triple croche ou 0,5 unité pour le $\mathrm{Fa}$ qui correspond à $\mathrm{U}$ et une croche pointée ou 2,5 unités pour le La qui correspond à $\mathrm{Ti}$ ). La hauteur des notes participe, parallèlement au rythme, à l'établissement d'une correspondance entre musique et langage : le changement d'intensité des syllabes est souligné par des intervalles ascendants et la succession de syllabes employant les mêmes voyelles est reproduite en gardant la même hauteur musicale.

Voyons le cas de la pièce appelée Kutsagükuegü (fig.5).

Fig.5.

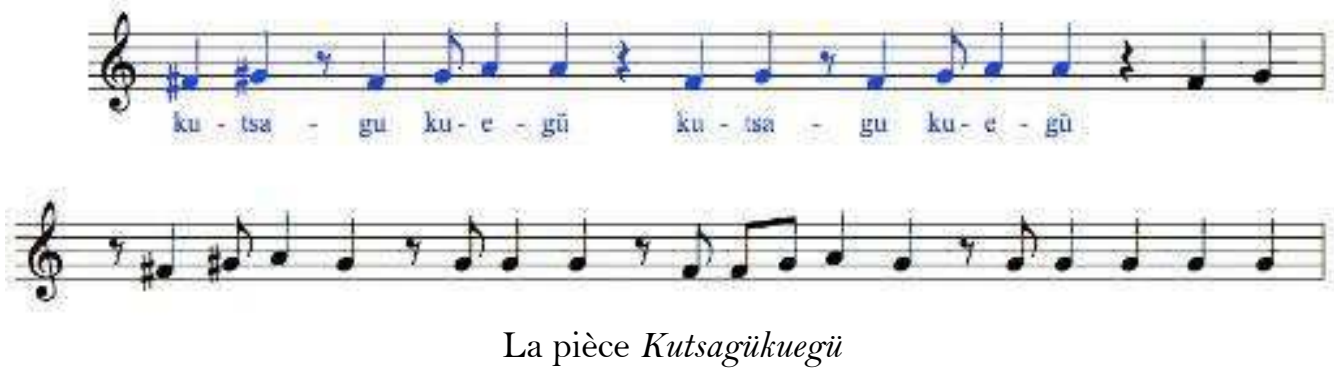

Sont reproduites en bleu les notes qui correspondent au nom de l'esprit : encore une fois il est utile de rappeler que le nom Kutsagükuegü, tout comme le mot Utigi, n'est pas

11 Cette analyse est tirée de «Musique et langage chez les Kuikuro du Haut Xingu » de Bruna Franchetto et Tommaso Montagnani, texte présenté le 20 et le 27 janvier 2010 dans le cadre du séminaire de Carlo Severi, L'image rituelle : énonciation, croyance, mémoire sociale, EHESS, Musée du Quai Branly. 
prononcé par une personne, mais entendu par les musiciens et par l'auditoire dans le son de la flûte. Le nom de l'esprit est ici «chanté» à deux reprises par la flûte (Audio.2, en Annexe): le profil mélodique de la deuxième occurrence du nom est très proche de la structure rythmique du mot Kutsagükueg ̈̈̈ ${ }^{12}$ (fig.6).

Fig.6.

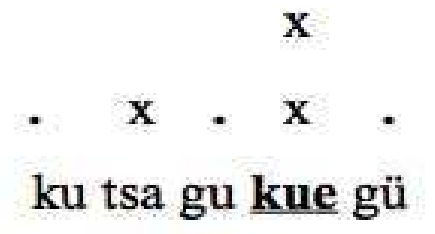

Profil du mot Kutsagükuegü

On observe un premier pic d'intensité sur la syllabe -Tsa- (un seul X dans l'analyse prosodique de la fig.6) : dans la mélodie, on constate que la note correspondante est un demiton au-dessus de celle qui la précède et de celle qui la suit (qui correspondent à $\mathrm{Ku}$ - et -Gu-). Le pic d'intensité le plus important (les deux X dans l'analyse prosodique de la fig.6), la syllabe -Kue-, est reproduit musicalement par deux notes, Sol et La : la deuxième note est la plus aiguë du fragment mélodique de Kutsagükuegü. La succession de croche/noire (donc une valeur rythmique brève suivie par une valeur plus longue) est un processus rythmique souvent utilisé dans la musique des flûtes pour recréer musicalement l'effet de l'accent tonique du mot.

À partir de ces deux analyses, voyons donc plus en détails quels sont les procédés musicaux qui participent au processus d'imitation du langage 13 par la musique. On peut observer fondamentalement cinq procédés qui permettent rapprocher le jeu de la flûte de la langue parlée : l'allongement de la durée, l'intervalle ascendant, les différences de hauteur, l'acciaccatura, l'attaque.

- À l'emphase de l'accent tonique du nom correspond un allongement de la durée de la note de la mélodie; ce procédé concerne les aspects rythmiques de la musique de Kagutu. Pour observer cela dans la pièce citée en exemple, Utigi, il suffira de voir sur la portée que la note de la mélodie qui correspond à -Ti- dans le nom de l'esprit est la plus longue des trois. C'est aussi le cas de la note la plus aiguë dans le passage qui correspond à la syllabe -Kue- dans la pièce Kutsagükuegü: la sensation de déplacement rythmique donnée par la noire qui suit la croche imite l'effet de l'accent.

- L'intervalle ascendant des notes peut dans certains cas s'ajouter à l'effet rythmique de l'allongement pour reproduire l'effet de l'accent tonique du nom : on est ici dans le domaine de la mélodie. Dans l'exemple de Utigi, la note la plus longue est aussi la plus aiguë dans l'intervalle Fa-La. On remarquera que l'accent tonique de la langue est reproduit en musique à la fois par un procédé rythmique (allongement) et mélodique (intervalle ascendant). En ce qui concerne la pièce Kutsagükuegü, on observe un premier intervalle ascendant sur la syllabe -Tsa- qui correspond au premier pic d'intensité du mot. Les notes qui correspondent au plus grand pic d'intensité du mot (-Kue-) sont les plus aiguës du fragment mélodique.

- Les écarts de hauteur dans la mélodie sont souvent utilisés dans les pièces Kagutu pour reproduire musicalement les différences entre voyelles graves et aiguës. Dans le fragment de mélodie qui correspond au nom Utigi, par exemple, les syllabes Ti et Gi sont reproduites par deux La, qui se situe une tierce majeure en dessus du Fa représentant la syllabe U. Le U ('ou') est une voyelle postérieure, arrondie, grave, tandis que le son I est antérieur, non 
arrondi, aigu. Il s'agit donc ici de l'établissement d'une relation entre un principe mélodique et les particularités phonétiques des noms des esprit : dans la mélodie, une note suivie par deux notes plus aiguës reproduit la succession d'un son grave et de deux sons aigus dans le nom.

- Des effets rythmiques reproduisent en musique la qualité des consonnes de la langue kuikuro : l'acciaccatura (une petite note d'agrément frappée très rapidement devant une note principale) est utilisée lorsque la flûte «prononce » le 'g' de Utigi, qui est un flap uvulaire typique de la langue kuikuro et qui n’a jusqu'à présent été observé dans aucune autre langue. Le flap uvulaire peut être réalisé phonétiquement comme occlusive vélaire ou fricative vélaire sonore, selon les voyelles contiguës.

- Un bref soupir permet d'obtenir une attaque plus nette dans la note qui suit immédiatement, et d'en accentuer la qualité percussive : c'est de cette façon que le son de la flûte reproduit certaines consonnes occlusives sourdes, comme le double 't' de Utigi.

\section{L’image de la pièce}

La musique kuikuro est basée sur l'alternance de deux types de phrase musicale : iina ou 'base' et itsikungu, la 'rupture'. L'alternance de ces deux parties est un facteur structurant de toute la musique kuikuro, de la même manière que dans les prières chamaniques ${ }^{14}$. Il est donc nécessaire que la différence entre les deux parties soit vite comprise par l'élève : les maîtres flûtistes recourent à une image pour permettre à l'élève de visualiser la structure de la pièce et la succession des parties. Pendant les recherches de terrain menées par Carlos Fausto 15 et par moi-même, cette image a été observée dans deux versions différentes. Je vais ici montrer le fonctionnement de ce système d'alternance base/rupture dans le contexte des suites de flûte Kagutu.

La 'base' est la phrase (ou les phrases) par laquelle la pièce commence. Ses caractéristiques principales sont d'une part une certaine régularité du rythme et du tempo, d'autre part l'utilisation du registre grave de la flûte.

La 'rupture' arrive en général vers le milieu de la pièce (plus rares sont les cas de pièces commençant par la 'rupture'). Elle se caractérise par une exécution en rubato (l'interprète prend beaucoup de liberté par rapport au rythme et au tempo de la 'base') et par l'utilisation du registre aigu de la flûte.

Une fois que la rupture a fait son apparition, le flûtiste expose à nouveau les motifs de base. Un certain nombre de pièces possèdent une deuxième ou une troisième rupture qui sont en général une variation ou un développement de la première. Dans l'image que les musiciens visualisent pour apprendre et exécuter la pièce, la rupture est conçue comme une déviation par rapport à un chemin droit (la base). Cela paraît évident en observant les deux dessins réalisés par le flûtiste Jakalu pendant les entretiens avec Carlos Fausto (fig.7) et avec moi-même (fig. 8).

14 Les Kuikuro opèrent une distinction entre chants et prières. La différence entre les chants et les prières n'est souvent pas définissable à partir de principes musicaux ; elle est plutôt basée sûr la fonction rituelle du texte et sur le contexte dans lequel il est utilisé.

15 C. Fausto, B. Franchetto, T. Montagnani, «Les formes de la mémoire. Art verbal et musique chez les Kuikuro du Haut-Xingu », L'Homme 197 (janvier 2011). Carlos Fausto est professeur à l'Université Fédérale de Rio de Janeiro. Il dirige le projet de recherche Documenta Kuikuro (DKK), dont le but est la documentation des rituels, des arts de la parole et des chants kuikuro. Le projet DKK a commencé en 2002 et se poursuit encore aujourd'hui. 


\section{Fig.7.}

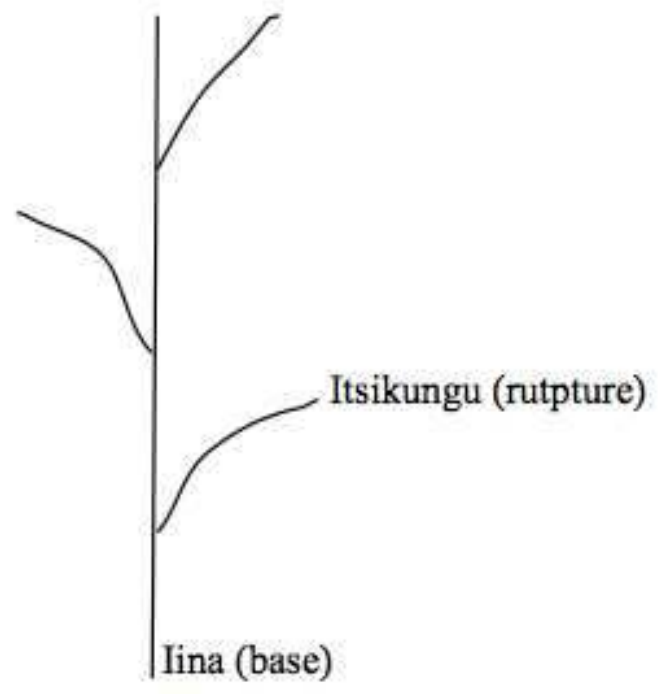

Jakalu Kuikuro explique iina et itsikungu à Carlos Fausto ${ }^{16}$

La ligne droite est donc la base, les ramifications sont les ruptures, dans lesquelles l'interprète ne s'engage que de manière temporaire : il devra ensuite revenir à la base et continuer le «droit chemin » jusqu'à la rupture suivante. Une seule pièce peut donc contenir plusieurs ruptures. Le dessin que Jakalu a fait pour moi lors de mon premier séjour de terrain montre la différence entre deux ruptures à l'intérieur d'une même pièce (fig.8).

\section{Fig.8.}

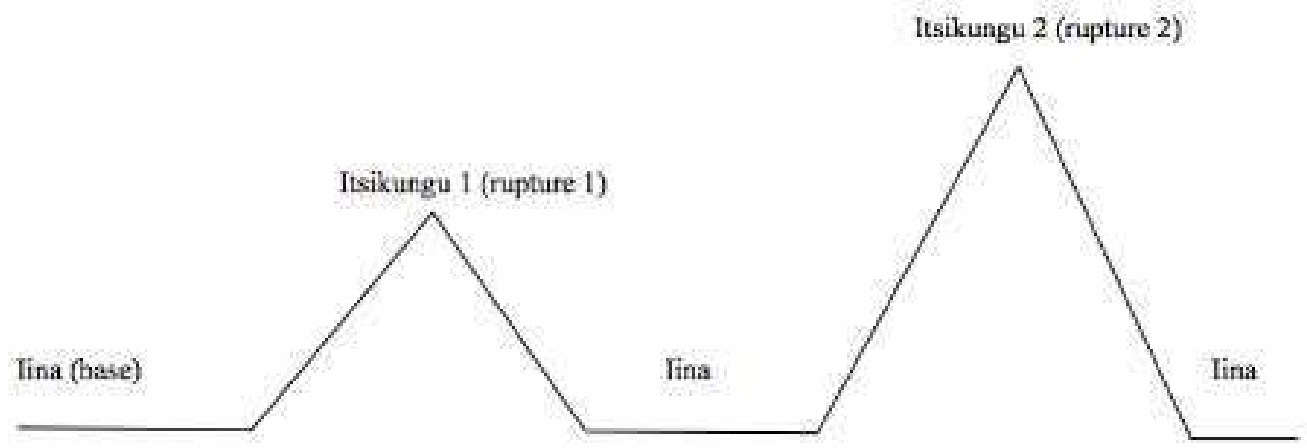

Jakalu Kuikuro explique iina et itsikungu à Tommaso Montagnani

Cette idée de montée et de descente est aussi présente dans les gestes que les musiciens (Jakalu et Tütekuegü) effectuent avec la main pendant la version vocale des pièces Kagutu. L'exécution vocale est en réalité la forme sous laquelle la musique de flûte sacrée est la plupart du temps entendue. Lorsque les musiciens exécutent les suites dans un contexte d'apprentissage ou dans un entretien avec les ethnologues, ils n'utilisent que très rarement la flûte (et lorsque c'est le cas, ils ne jouent pas de l'instrument rituel, mais d'une petite flûte d'apprentissage) : ils se servent d'une technique vocale où des syllabes remplacent le son de l'instrument. N'ayant pas à manier un instrument, les musiciens accompagnent avec leurs mains le développement mélodique au moyen de gestes très codifiés. Pour la base, le musicien bouge sa main au-dessous de ses genoux, la paume tournée vers le sol, en effectuant des mouvements latéraux, comme pour donner l'idée d'une surface plaine et basse. Quand la 
première rupture arrive, il lève d'abord son index et ensuite son bras vers le haut : au milieu de la rupture sa main, l'index levé pointant vers le haut, est au-dessus de sa tête. À la fin de la rupture, l'index commence à pointer vers le bas, le musicien baisse son bras et revient au geste initial en même temps que son chant revient à la base.

En écoutant la musique Kagutu on peut constater qu'il existe une correspondance entre ces dessins, les gestes de la main et les changements de hauteurs des notes dans la pièce : les phrases itsikungu (ruptures) sont en effet plus aiguës que les phrases iina (base). En revanche dans les pièces avec plusieurs ruptures, celles qui arrivent après la première ne sont pas plus aigues, comme le deuxième dessin pourrait le laisser croire. Jakalu dessine itsikungu 2 avec une courbe plus élevée parce que cette phrase dure plus longtemps qu'itsikungu 1. Les valeurs rythmiques sont encore plus dilatées et le phrasé rubato est accentué. Cette idée de montée exprimée dans le dessin et dans les gestes est donc basée à la fois sur la hauteur des notes et sur leur durée. Nous avons une courbe plus haute pour itsikungu 1 parce que la mélodie monte, mais la raison pour laquelle la courbe d'itsikungu 2 est encore plus élevée est sa durée dans le temps, et ainsi la sensation d'un éloignement encore plus grand. L’idée d’une plus grande hauteur exprimée par le signe graphique est associée, en musique, à des principes à la fois mélodiques et rythmiques.

Cette opposition entre les deux parties est non seulement essentielle au développement de la pièce mais elle garantit aussi l'homogénéité stylistique des pièces appartenant à une même suite. Toutes les pièces appartenant à la suite dénommée Titalo, par exemple, partagent les mêmes phrases de 'base' (ou du moins ces phrases sont très similaires d'une pièce à l'autre). Ce qui différencie les pièces appartenant à une même suite et qui constitue leur marque identitaire sont les ruptures : chaque pièce a ses propres 'ruptures'. Itsikungu est considérée comme la partie la plus importante de la pièce musicale car elle permet son identification.

\section{Le parcours de Kagutu : cartes mentales et mouvements dans l'espace}

L'image engendre donc la musique. Nous avons montré la façon dont l'image contribue à l'organisation de la pensée musicale chez les maîtres kuikuro. La visualisation mentale de formes et de mouvements offre un support sur lequel les musiciens reproduisent et déploient les structures de la pièce musicale. La musique à son tour engendre de nouvelles images, des cartes mentales auxquelles correspondent des mouvements des musiciens dans l'espace. La musique des flûtes Kagutu est subdivisée en suites. Chaque suite compte de 15 à 50 pièces: nous avons vu dans le paragraphe précédent la façon dont chaque pièce est structurée et « visualisée » mentalement. L'idée d'un chemin principal et d'une ou plusieurs déviations qui est à la base de la conception de la structure interne de la pièce est aussi utilisée par les musiciens pour reconstruire la succession des suites pendant le rituel. Dans le schéma qui suit, j’ai essayé de représenter la succession des suites telle qu'elle m’a été expliquée par Tütekuegü Kuikuro, l'un des mes informateurs. Le schéma (fig.9) montre le rituel Kagutu exécuté par deux trios de flûtistes : un trio qui joue de flûtes Kagutu et un autre qui se sert de flûtes Kuluta, plus petites et en bambou. Le rituel Kagutu peut être réalisé par un seul trio (dans ce cas toujours des flûtes Kagutu) ou par les deux. 
Fig.9.

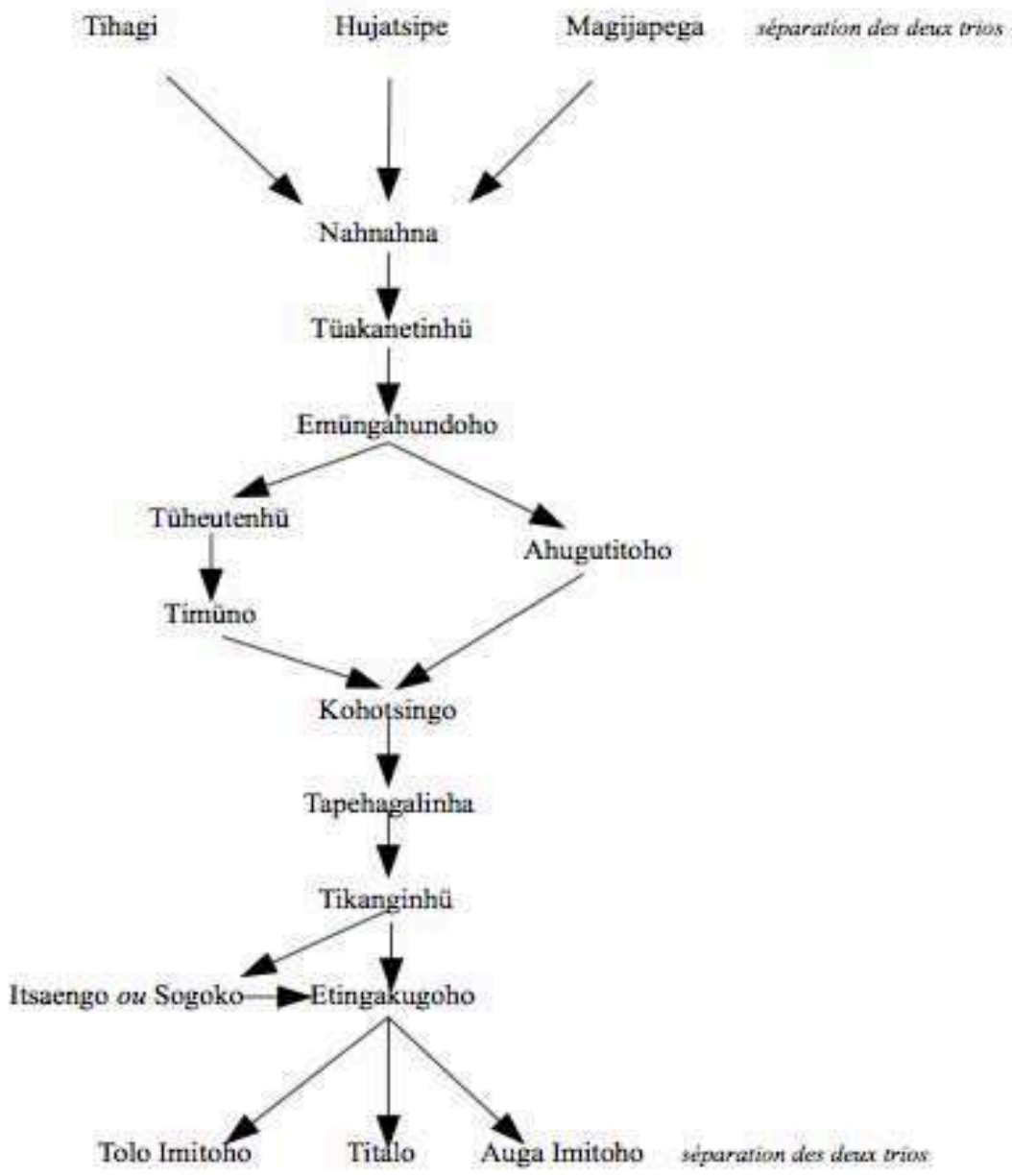

Le rituel Kagutu exécuté par deux trios de flûtistes

Les noms sur le schéma sont ceux des suites Kagutu. L’idée de parcours qui caractérise la musique Kagutu au niveau de la microstructure (la structure interne de la pièce, base et ruptures) est aussi présente au niveau de la macrostructure, dans la conception du répertoire dans sa totalité. Dans son explication, Tütekuegü a utilisé des expressions et des termes similaires à ceux dont il s'était servi pour illustrer les concepts de iina et de itsikungu. Par exemple : « là, on continue tout droit, ensuite on va par là (en indiquant à droite ou à gauche avec sa main, NDA) et puis on revient et on continue tout droit » ou «maintenant tu peux aller par là (à droite) ou par là (à gauche) ». La succession des suites est conçue comme un chemin droit au cours duquel un certain nombre de « déviations » sont autorisées. Ces déviations doivent elles aussi suivre des parcours secondaires fixes et, surtout, il est impératif de revenir au chemin principal après les avoir empruntées. L'exécuteur imagine cette séquence comme un parcours à l'intérieur duquel il a la possibilité de changer de route à certains endroits préétablis.

Le rituel Kagutu peut être joué par les seules flûtes Kagutu ou par un trio de flûtes Kuluta qui les accompagnent. Le schéma de la succession des suites peut donc être lu de deux façons différentes. Au début et à la fin, on peut observer trois suites sur la même ligne : lorsque le rituel est exécuté sans les Kuluta, les flûtistes Kagutu choisissent une des trois suites possibles et passent à la suivante. Lorsque les flûtistes Kuluta participent au rituel, deux des trois suites présentes sur la même ligne au début de la séquence (Tihagi, Hujiatsipe, Magijapega) sont jouées en même temps, l'une par les musiciens Kuluta et l'autre par les Kagutu. Il y a donc là une «séparation » entre les deux trios, qui se rejoignent et jouent la 
même chose sur les suites qui suivent. Cette «séparation » du début existe seulement au niveau de la musique et de la carte mentale, puisque les deux trios de flûtistes jouent dans le même espace, la maison centrale (Kuakutu). En revanche, à la fin de la séquence, cette séparation devient physique: chacun des deux trios choisit une des trois suites possibles (Tolo Imitoho, Titalo, Auga Imitoho) et commence à sortir de l'espace rituel : le trio Kagutu commence à jouer en dansant et en marchant dans une direction, tandis que le trio Kuluta fait la même chose dans la direction opposée, en jouant une suite différente de celle choisie par le trio Kagutu. Les deux trios effectuent un tour du village et passent devant chaque maison (fig. 10).

Fig.10.

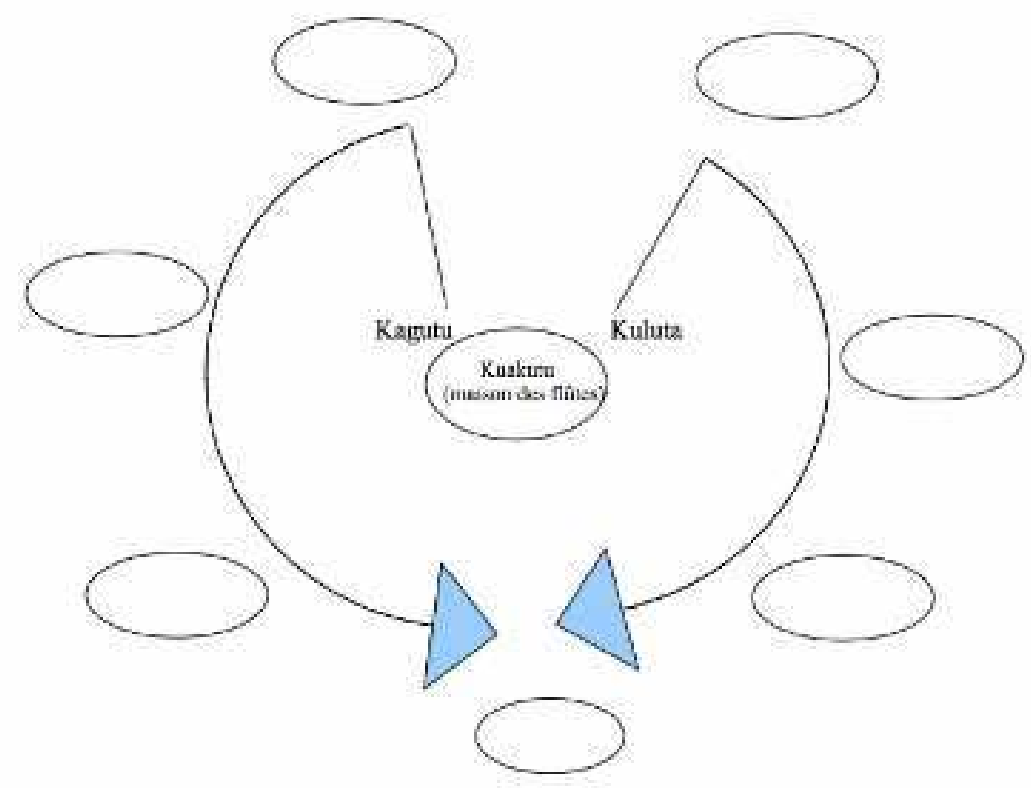

Tour du village par les trios Kagutu et Kuluta, chacun ayant choisi l'une des trois suites possibles à la fin de la séquence (Tolo Imitoho, Titalo, Auga Imitoho)

À l'intérieur de la séquence/parcours, on observe une première bifurcation possible après la suite Emüngahundoho: les flûtistes peuvent exécuter soit Ahugutitoho soit Tuheutenhü: lorsqu'ils optent pour cette deuxième possibilité ils sont obligés d'exécuter Timüno avant de pouvoir revenir au «chemin principal» et continuer le parcours avec la suite Kohotsingo. Il convient ici de rappeler que le parcours en question est mental et musical, les flûtistes n'étant pas encore sortis de la maison centrale. Après la suite Tikanginhü, un autre choix se présente aux musiciens : soit ils continuent tout droit et jouent Etingakugoho, soit ils font un détour et jouent Itsaengo, la suite la plus difficile et dangereuse. Itsaengo est rarement exécutée et uniquement lorsque le maître du rituel (la personne qui demande de l'effectuer) souhaite célébrer un événement important. Arrivés à la fin du parcours mental et musical, les musiciens commencent donc à bouger et effectuent les déplacements et la séparation des deux trios que je viens de décrire.

\section{Considérations finales}

Le système musical kuikuro emploie plusieurs types d'images à la fois pendant la phase d'apprentissage et lors de l'exécution rituelle des répertoires de flûte Kagutu. Des 
images sonores (une mélodie de flûte qui reproduit un mot qui est un esprit) et des images mentales, des formes et des parcours, qui servent de support mnémonique à l'exécution. Dans la mémoire de l'interprète le son occupe des espaces mentaux et s'y orientent : à un moment donné de l'exécution, l'espace de la mémoire musicale coïncide avec l'espace physique du rituel. Les mouvements de la musique dans le parcours mental de la succession des suites trouvent une correspondance avec les mouvements des flûtistes dans l'espace réel. Les deux trios de flûtes prennent deux directions différentes au moment où le parcours mental de la succession des suites propose plusieurs chemins possibles. Cette correspondance entre le mouvement dans l'espace et la succession des suites de musique à jouer est un exemple de la façon dont la connexion entre musique et image est établie chez les Kuikuro.

Il me semble que dans le système musical de ce groupe xinguanien, il est important de considérer les trois éléments (musique, langage et image) comme indissociables et nécessaires au processus de figuration de l'invisible. L'énonciation de noms propres au moyen du son de la flûte, la visualisation d'images, l'exécution des pièces et les mouvements des flûtistes, tout cela doit être considéré dans sa totalité comme un unique processus de figuration de l'invisible. Bien qu'il s'agisse d'un processus se faisant en deux temps, puisque la musique doit être d'abord apprise par les musiciens et ensuite exécutée pour l'auditoire, le rapport aux images et aux noms garantit une continuité entre les deux phases.

Plusieurs niveaux d'invisibilité, qui progressivement se font perceptibles tout au long de la performance rituelle, coexistent dans la phase d'apprentissage et dans l'exécution rituelle des musiques Kagutu : les esprits deviennent audibles par le son, le son à son tour est rendu visible par les gestes avec lesquels les flûtistes accompagnent leur exécution vocale. Ces gestes, qui ne sont exécutés qu'en contexte d'apprentissage ou d'entretien ethnographique, correspondent à un autre schéma, qui n'est partagé que par les musiciens flûtistes sous forme d'image mentale (le chemin avec les déviations, la base et la rupture). L'auditoire est d'habitude en mesure de reconnaître la différence entre les parties base et rupture (les rythmes et le profil mélodique changent de façon considérable), mais il ne sait pas les nommer et ne connaît pas la structure de la pièce, il ne maîtrise pas la séquence des parties ni l'ordre des pièces à l'intérieur d'une suite ou des suites pendant le rituel. L'image composée par les mouvements des flûtistes dans l'espace correspond en partie à la forme sous laquelle le répertoire est visualisé et mémorisé par les musiciens. Il s'agit dans ce cas d'une figuration différentielle, puisque l'image complète du «parcours » des suites n'est connue que par les flûtistes. Néanmoins, l'exécution des suites Kagutu transfère sur le plan auditif un outil mnémonique de type visuel.

L'apprentissage et l'exécution de la musique des flûtes Kagutu sont donc basés sur un équilibre, d'une part entre ce qui est partagé par la communauté et ce qui est conservé par des initiés (les musiciens) et d'autre part entre les multiples formes de manifestation de ce qui ne peut pas être vu dans sa forme originelle.

Tommaso Montagnani a été allocataire de recherche à l'EHESS de 2006 à 2009 et il est actuellement boursier au Musée du Quai Branly. Il a mené deux enquêtes de terrain chez les Kuikuro du Haut Xingu en 2008 et en 2009. Il a participé au projet de documentation Documenta Kuikuro (DKK), dirigé par Carlos Fausto et Bruna Franchetto (Universidade Federal de Rio de Janeiro) et effectué en collaboration avec des chercheurs indigènes. Il s'intéresse aux analogies structurelles entre les arts de la parole et la musique instrumentale Kuikuro. 